\title{
Density of states in d-wave superconductors disordered by extended impurities
}

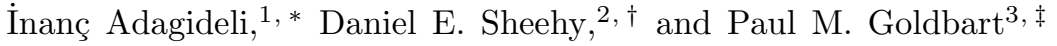 \\ 1 Instituut-Lorentz, Universiteit Leiden, Niels Bohrweg 2, Leiden, NL-2333 CA, The Netherlands \\ 2 Department of Physics \& Astronomy, Univ. of British Columbia, \\ 6224 Agricultural Rd., Vancouver, B.C. V6T1Z1, Canada \\ 3 Department of Physics, University of Illinois at Urbana-Champaign, \\ 1110 West Green Street, Urbana, Illinois 61801, U.S.A.
}

(Dated: January 18, 2002)

\begin{abstract}
The low-energy quasiparticle states of a disordered d-wave superconductor are investigated theoretically. A class of such states, formed via tunneling between the Andreev bound states that are localized around extended impurities (and result from scattering between pair-potential lobes that differ in sign) is identified. Its (divergent) contribution to the total density of states is determined by taking advantage of connections with certain one-dimensional random tight-binding models. The states under discussion should be distinguished from those associated with nodes in the pair potential.
\end{abstract}

PACS numbers: 74.25.-q, 74.72.-h, 74.25.Jb

Introduction: In recent years, considerable attention has been focused on the low-energy electron-hole quasiparticle spectral properties of the cuprate superconductors in the presence of impurity scattering. Much of the impetus for this effort has its origin in the fact that many of the cuprate superconductors are randomly chemically doped insulators, and are therefore disordered. Moreover, as they are pair-breakers for them, the role of impurities is especially important for d-wave superconductors. Of particular interest is the behavior of the single-particle density of states (DOS) $\rho(E)$ as the energy $E$ tends to zero, i.e., its low-energy behavior.

In recent work on the DOS of disordered d-wave superconductors, Pépin and Lee invoked a $t$-matrix approximation to infer that $\rho(E) \sim 1 /\left.E \ln ^{2}\right|^{2}$ at loy energies. More recently, Yashenkin et al. ${ }^{2}$ and Altland 1 have argued that the divergence found in Ref. is present only for the case of a vanishing chemical potential (i.e. for a halffilled band), and thus does not apply to a doped cuprate. (To be precise, unitarity of the impurity scattering is also required.) It is further argued in Refs. 2.3 that, instead of diverging, $\rho(E)$ should vanish at $E=0$ (unless certain very specific fine-tuning requirements are met). An important feature shared by Refs. 1 . 2.3 is the hypothesis that the disorder potential may be adequately modeled by a random collection of point-like scatterers. However, for a single impurity in a d-wave superconductor, the lowenergy DPS is qualitatively different for point-like 4 and extended (i.e. impurities of a size much larger than the Fermi wavelength) impurities: the states that reside at zero energy for extended impurities reside at nonzero energies for point-like impurities. (The underlying reason for this difference is that for point-like impurities, the quasiparticle scattering is essentially diffractive, whereas for the extended impurities it is essentially semiclassical.) This observation raises the possibility that such differences will continue to manifest themselves in the many-impurity setting.

The purpose of the present Paper is to identify a mech- anism for producing low-energy quasiparticle states. This mechanism is based on impurity-scattering processes that connect states associated with differing signs of the dwave pair potentiall. In the case of a single impurity this mechanism has already been shown to produce lowenergy states that are localized near the impurity. These states can be associated with the classical trajectories scattering from the impurity, andhave been observed via scanning-tunneling spectroscopy相.

Here, we build upon this single-impurity physics to identify a singular (and potentially dominant) contribution to the low-energy DOS. This contribution, which conventional techniques fail to capture, arises from tunnelling along the classical trajectories that connect the individual impurities and, hence, connect the low-energy states localized near these impurities. The underlying physics was formulated some time ago in the context of tunnelling corrections to ground-state energies in in models of supersymmetric quantum mechanics 91212.

The picture we have in mind(see Fig. 1) of the processes that lead to low-energy states involves classical trajectories that each visit many extended impurities. As a quasiparticle progresses along such a trajectory, its momentum is repeatedly altered via scattering from the extended impurities, so that the effective pair-potential (Fig. 1]b) undergoes sign changes. Localized near each such sign-change would be a zero-energy quasiparticle state; quasiparticle tunnelling (through the pair-potential) connects these states, raising their energies from zero, and thus forming a low-energy band that exhibits a Dyson-like singularity 12 at zero energy: $\rho(E) \sim 1 / E\left|\ln E^{2}\right|^{3}$. This picture loses its precision for sequences of impurities between which the pair potential is predominantly small (i.e. for nodal directions) and, consequently, the states are not well localized near the impurities. However, the contribution on which we are focusing (i.e. the non-nodal contribution) is expected to be substantial, and quite likely dominant, in the lowenergy limit. 


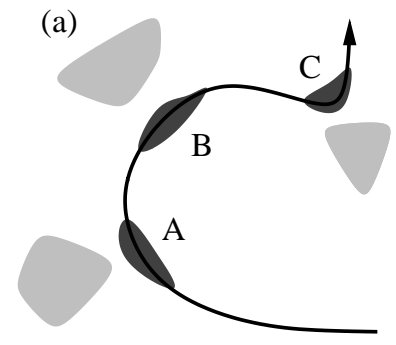

(b)
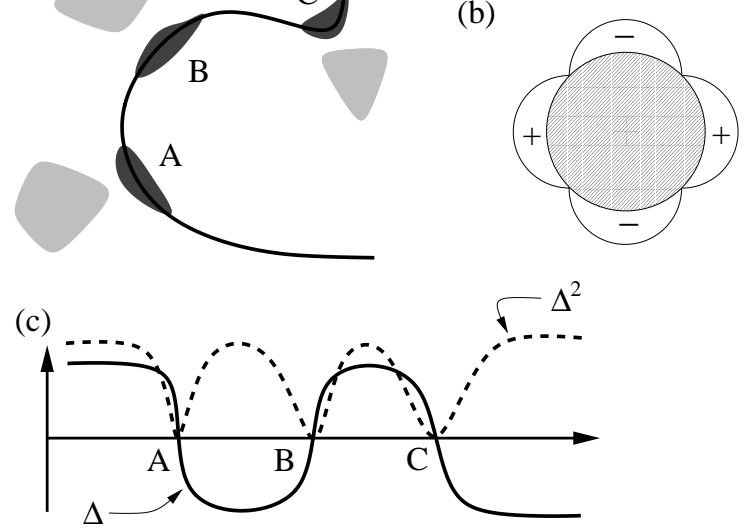

FIG. 1: (a) Sketch of a classical trajectory that encounters several impurities and, hence, several sign-changes in $\Delta$. The shaded regions on the trajectory denote the sections of the trajectory where approximate zero-energy states reside. (b) Momentum-space pair potential of a d-wave superconductor. (c) Schematic depiction of $\Delta$ and $\Delta^{2}$ along the trajectory.

Basef on previous work on low-energy quasiparticle statest 5 , we identify three classes of processes that can effect the low-energy DOS of a disordered d-wave superconductor: (i) purely semiclassical scattering between states with differing signs of the pair-potential (i.e. scattering due to random extended scatterers in d-wave superconductors), which we shall focus on in the present Paper; (ii) purely diffractive scattering between states near the nodal directions of the pair-potential (i.e. the scattering of nodal quasiparticles by point-like impurities), which was considered in, e.g., Refs.2.313; and (iii) the mixing of the low-energy states that arise from processes (i) and (ii). Throughout the rest of this Paper we shall ignore processes in class (iii). In consequence, the low-energy DOS of a disordered d-wave superconductor can be expressed as a sum of contributions arising from class (i) processes (viz. $\rho_{\text {ext }}$ ) and class (ii) processes (viz. $\rho_{\text {nodal }}$ ), the latter, as discussed in Refs. 2 , being non-divergent and therefore subdominant. Thus, we shall focus on $\rho_{\text {ext }}$ [and denote this by $\rho(E)$ ].

Quasiparticle motion in a $d$-wave superconductor with many extended impurities: Our focus will be on the DOS per unit area, i.e.,

$$
\rho(E) \equiv \frac{1}{A} \sum_{m} \delta\left(E-E_{m}\right),
$$

where $A$ is the area of the sample and the energy eigenvalues $\left\{E_{m}\right\}$ follow from the Bogoliubov-de Gennes eigenproblem, viz.,

$$
\left(\begin{array}{cc}
\hat{h} & \hat{\Delta} \\
\hat{\Delta} & -\hat{h}
\end{array}\right)\left(\begin{array}{l}
u_{n} \\
v_{n}
\end{array}\right)=E_{n}\left(\begin{array}{l}
u_{n} \\
v_{n}
\end{array}\right)
$$

Here, $\hat{h} \equiv-\nabla^{2}-k_{\mathrm{F}}^{2}+V(\mathbf{r})$, in which $k_{\mathrm{F}}^{2}$ is the chemical potential [i.e. $k_{\mathrm{F}}\left(\equiv 2 \pi / \lambda_{\mathrm{F}}\right)$ is the Fermi wave vector], $V$ is the single-particle impurity potential, and we have adopted units in which $\hbar^{2} / 2 m=1, m$ being the common (effective) mass of the electrons and holes. The operator $\hat{\Delta}$ is the pair-potential (integral) operator; how it acts is specified by the nonlocal pair-potential kernel $\Delta\left(\mathbf{r}, \mathbf{r}^{\prime}\right)$ via $\left[\hat{\Delta} v_{n}\right](\mathbf{r})=\int d^{2} r^{\prime} \Delta\left(\mathbf{r}, \mathbf{r}^{\prime}\right) v_{n}\left(\mathbf{r}^{\prime}\right)$.

To define this model fully, we need an assumption about the form of $\Delta\left(\mathbf{r}, \mathbf{r}^{\prime}\right)$. It is convenient to exchange the coordinates $\mathbf{r}$ and $\mathbf{r}^{\prime}$ for the relative and center-ofmass coordinates $\boldsymbol{\rho}$ and $\mathbf{R}$ :

$$
\bar{\Delta}(\boldsymbol{\rho}, \mathbf{R}) \equiv \Delta\left(\mathbf{r}, \mathbf{r}^{\prime}\right), \quad \boldsymbol{\rho} \equiv \mathbf{r}-\mathbf{r}^{\prime}, \quad 2 \mathbf{R} \equiv \mathbf{r}+\mathbf{r}^{\prime}
$$

Then, via Fourier transformation with respect to $\boldsymbol{\rho}$, viz.,

$$
\bar{\Delta}(\mathbf{k}, \mathbf{R}) \equiv \int d^{2} \rho \mathrm{e}^{-i \mathbf{k} \cdot \boldsymbol{\rho}} \bar{\Delta}(\boldsymbol{\rho}, \mathbf{R})
$$

we obtain the pair-potential $\bar{\Delta}(\mathbf{k}, \mathbf{R})$ at center-of-mass position $\mathbf{R}$ and relative momentum $\mathbf{k}$. As our aim is to describe the cuprate superconductors, we take $\bar{\Delta}(\mathbf{k}, \mathbf{R})$ to have d-wave symmetry: $\bar{\Delta}(\mathbf{k}, \mathbf{R}) \propto\left(k_{x}^{2}-k_{y}^{2}\right)$, where $k_{x}$ and $k_{y}$ denote the cartesian components of $\mathbf{k}$. However, we allow for the possibility of position-dependent amplitude variations of the d-wave pair-potential due, say, to pair-breaking effects near the extended scatterers.

Semiclassical approach to the Bogoliubov-de Gennes eigenproblem: We now invoke a semiclassical approximation under which $\rho(E)$ is expressed in terms of the solution of a family of one-dimensional eigenproblems, each associated with a classical scattering trajectory in the presence of the single-particle impurity potential $V(\mathbf{r})$. We restrict ourselves to a brief discussion of this approach; for details see Refs 14 . The approximation amounts to our: (i) regarding the kinetic and potential energies as being comparable and being the largest energies in the problem; (ii) turning off the pair potential; (iii) treating semiclassically the quasiparticle motion in the presence of the kinetic and potential energies; and (iv) reinstating the pair potential. Via this approach, we reduce the two-dimensional Bogoliubov-de Gennes eigenproblem to a family of one-dimensional Andreev eigenproblems residing on trajectories, each trajectory being a particular classical scattering trajectory in the presence of the many-impurity potential. This scheme applies under the following conditions: (i) the amplitude of $\bar{\Delta}$ and $V$ should vary slowly, relative to the Fermi wavelength $\lambda_{\mathrm{F}}$; and (ii) the Fermi energy $k_{\mathrm{F}}^{2}$ should be large compared with the energy scale of interest, viz. $E$, as well as with the typical pair-potential scale.

Let us now turn to the family of one-dimensional eigenproblems arising from this semiclassical scheme. Following Ref.l, these trajectory-dependent eigenproblems take 
the form

$$
\begin{aligned}
& \hat{H}\left(\begin{array}{c}
\bar{u}_{n} \\
\bar{v}_{n}
\end{array}\right)=E_{n}\left(\begin{array}{c}
\bar{u}_{n} \\
\bar{v}_{n}
\end{array}\right), \\
& \hat{H} \equiv\left(\begin{array}{cc}
-2 i k_{\mathrm{F}} \partial_{\sigma} & \Delta_{0}(\sigma) \\
\Delta_{0}(\sigma) & 2 i k_{\mathrm{F}} \partial_{\sigma}
\end{array}\right), \\
& \Delta_{0}(\sigma) \simeq \bar{\Delta}\left(k_{\mathrm{F}} \partial_{\sigma} \mathbf{x}_{\mathrm{c}}(\sigma), \mathbf{x}_{\mathrm{c}}(\sigma)\right),
\end{aligned}
$$

i.e., an Andreev eigenproblem 15 . Here, the parameter $\sigma$ measures the position along a particular classical trajectory $\mathbf{x}_{\mathrm{c}}(\sigma)$, the latter obeying Newton's equation in the many-impurity potential, viz.,

$$
k_{\mathrm{F}}^{2} \partial_{\sigma}^{2} \mathbf{x}_{\mathrm{c}}(\sigma)=-\nabla V\left(\mathbf{x}_{\mathrm{c}}(\sigma)\right) .
$$

The DOS is then obtained by assembling the eigenvalue spectra $E_{n}(\mathbf{n}, b)$ of all the classical trajectories, the latter being labelled in terms of an asymptotic momentum direction $\mathbf{n}$ and impact parameter $b$ :

$$
\begin{aligned}
\rho(E) & \simeq \frac{k_{\mathrm{F}}}{A} \int \frac{d \mathbf{n}}{2 \pi} \int d b \rho(\mathbf{n}, b, E), \\
\rho(\mathbf{n}, b, E) & \equiv \sum_{m} \delta\left(E-E_{m}(\mathbf{n}, b)\right) .
\end{aligned}
$$

Thus, in order to obtain $\rho(E)$ one needs to find each classical trajectory, obtain the associated effective pairpotential [given by Eq. (5c)], solve the resulting onedimensional eigenvalue equation and, finally, integrate over all the classical trajectories using Eq. (7a). We note that if one interprets the weight of a particular classical trajectory as the probability of finding a pair-potential configuration corresponding to that particular trajectory then we see that the calculation of $\rho(E)$ amounts to computing the average density of states of a random pairpotential model. Medelif of this sort have been considered, e.g., in Refs 16.17 .18 .19$.

Eigenvalue problem for a single trajectory: We now examine the contribution $\rho(\mathbf{n}, b, E)$ to the DOS for the case of a generic trajectory $(\mathbf{n}, b)$. For convenience, we introduce the rescaled trajectory parameter $s \equiv \sigma / 2 k_{\mathrm{F}}$; the Hamiltonian then becomes

$$
\hat{H}=\left(\begin{array}{cc}
-i \partial_{s} & \Delta(s) \\
\Delta(s) & i \partial_{s}
\end{array}\right),
$$

where $\Delta(s) \equiv \Delta_{0}\left(2 k_{\mathrm{F}} s\right)$.

Our method for calculating the spectrum of $\hat{H}$ in the many-impurity case is based on that for a single impurity $\bar{E}$. In the latter case, low-energy states arose from asymptotically sign-changing trajectories [i.e. those trajectories for which $\lim _{s \rightarrow \pm \infty} \Delta(s)$ differ in sign]. Finding the spectrum amounted to identifying such sign-changing trajectories. What about the case of many extended impurities? In this case, for a typical trajectory through the impurity potential $\Delta(s)$ undergoes repeated sign changes. On a particular trajectory let us label the the positions of these zeroes of $\Delta(s)$ by $\left\{s_{n}\right\}$. Recall that we are concerned with the collection of impurity states that would lie at zero energy if the impurities were isolated. Owing to tunnelling between them, these formerly degenerate states yield a continuum of states, extending upwards in energy from zero. Our task is to shed some light on this band-formation. We proceed to set up a tight-binding model along the trajectory, in which we retain only the zero-energy impurity states $\{|n\rangle\}$ (i.e. the local ground states at each of the $\left\{s_{n}\right\}$ ) anf allow only nearest-neighbor tunneling between themen 21. To complete the model, we need the matrix elements of $\hat{H}$ connecting these states, i.e.

$$
t_{n} \equiv\langle n|\hat{H}| n+1\rangle .
$$

Using the analytic expression for the zero energy wavefunctions 11 , $\langle s \mid n\rangle \propto \exp -\int_{s_{n}}^{s} d s^{\prime}\left|\Delta\left(s^{\prime}\right)\right|$, a straightforward calculation produces

$$
t_{n} \approx \frac{1}{\sqrt{\pi}}\left|\Delta^{\prime}\left(s_{n}\right) \Delta^{\prime}\left(s_{n+1}\right)\right|^{1 / 4} \exp \left\{-\int_{s_{n}}^{s_{n+1}} d s^{\prime}\left|\Delta\left(s^{\prime}\right)\right|\right\},
$$

where $\Delta^{\prime}(s) \equiv \partial_{s} \Delta(s)$. We are now in a position to write down a low-energy effective approximation to $\hat{H}$, viz.,

$$
\hat{H} \approx \sum_{n} t_{n}(|n\rangle\langle n+1|+| n+1\rangle\langle n|),
$$

i.e., for each classical trajectory, one arrives at a (topologically) one-dimensional hopping model that captures the physics of tunneling processes between the (formerly zero-energy) states localized near each zero of the pairpotential.

Density of states: In order to obtain the low-energy DOS, we must obtain the DOS of the effective Hamiltonian (11) for each trajectory, and then collect them together. We assume that the collection of trajectories forms an ensemble that is characterized by the condition that momentum directions before and after a collision are uncorrelated. Then, summing over such an ensemble of trajectories is equivalent (up to a constant of proportionality) to averaging the DOS of the Hamiltonian (11) over uncorrelated values of $t_{n}$. To obtain the low-energy DOS of this effective model ve appeal to results obtained by Eggarter and Riedinger22, who, building on the work of Dyson 12 and Theodorou and Cohen23, studied random-hopping models of precisely this form. Specifically, in Ref 22 it was found that, under the condition that the $\left\{t_{n}\right\}$ are uncorrelated 24 and identically distributed, the DOS as $E \rightarrow 0$ is given by

$$
\rho(E) \approx N_{\mathrm{s}} Z \frac{2 \sigma^{2}}{E\left|\ln (E / \bar{t})^{2}\right|^{3}},
$$

where $N_{\mathrm{s}}$ denotes the average number of sites along the trajectory, $Z$ is the constant of proportionality arising from the Jacobian of the transformation from summing over trajectories to averaging over $\left\{t_{n}\right\}, \bar{t}$ is the scale characterizing $\left\{t_{n}\right\}$, and the amplitude $\sigma^{2}$ is given by the variance of the logarithm of $t$, i.e.,

$$
\sigma^{2} \equiv\left\langle\left(\ln t^{2} / \Delta_{0}^{2}\right)^{2}\right\rangle-\left\langle\ln t^{2} / \Delta_{0}^{2}\right\rangle^{2}
$$


where $\langle\cdots\rangle$ denotes a disorder average. The scale $\bar{t}$ and $\sigma^{2}$ could be estimated with the help of Eq. 10 as

$$
\begin{aligned}
& \vec{t}^{2} \propto k_{\mathrm{F}} \Delta_{0} n_{\mathrm{c}}^{1 / 2} \exp \left(-\Delta_{0} / k_{\mathrm{F}} n_{\mathrm{c}}^{1 / 2}\right), \\
& \sigma^{2} \propto \Delta_{0} / k_{\mathrm{F}} n_{\mathrm{c}}^{1 / 2}
\end{aligned}
$$

where $n_{\mathrm{c}}$ is the number of impurities per unit area. What remains is to determine the coefficient $Z$; we now make an estimate of this quantity.

Our estimate for $Z$ follows from considering the integration over $b$ for a single impurity of size $a$. As the impurity potential is expected to decay rapidly away from the impurity, the only trajectories that interact appreciably with it are those that directly intersect it, i.e., $k_{\mathrm{F}} \int d b \rightarrow k_{\mathrm{F}} a$. Thus, in the absence of the tunneling corrections between the zero-energy states $\rho(E)$ is approximately given by $\rho_{0}(E) \approx k_{\mathrm{F}} a n_{\mathrm{c}} \delta(E)$. Although the inclusion of tunneling corrections changes the energy dependence of $\rho(E)$, here we assume that the energyintegrated density of states $\int_{0}^{\epsilon} \rho(E) d E$ is approximately conserved for some appropriately chosen cutoff $\epsilon$. These considerations lead to the following approximate form for $\rho(E)$ valid for $E \ll \bar{t}$ :

$$
\rho(E) \propto \frac{\Delta_{0} a n_{\mathrm{c}}^{1 / 2}}{E\left|\ln (E / \bar{t})^{2}\right|^{3}} .
$$

We remark that the divergence of the low-energy DOS is ultimately cut-off due to physical processes not included in the present description. These include the dephasing scale $\hbar / \tau_{\phi}$ and the diffractive scattering scale $\hbar / \tau_{\text {d.s. }}$; the cut-off will occur at the largest of these scales. Thus, it is possible that at extremely low energies $\rho(E)$ eventually does vanish asymptotically, in agreement with the results of Refs.2.3. However, the present results would still apply at intermediate energies. Finally, we stress that the calculation presented here does not place any special emphasis on the nodes of the d-wave order parameter; indeed, the singular contribution to the density of states of a d-wave superconductor reported here arises from states in generic (rather than nodal) regions on the Fermi surface.

Acknowledgments: PMG thanks Alexei Tsvelik for informative discussions and the University of Colorado at Boulder for its kind hospitality. This work was supported by the U.S. Department of Energy under Award No. DEFG02-ER9645439, through the Frederick Seitz Materials Research Laboratory, by the Dutch Science Foundation NWO/FOM (IA), and by NSERC (DES).
* Electronic address: adagidel@lorentz.leidenuniv.n

$\dagger$ Electronic address: sheehv@phvsics.ubc.ca

‡ Electronic address: goldbart@uiuc.edu

1 C. Pépin and P. A. Lee, Phys. Rev. B 63, 054502 (2001).

2 A. G. Yashenkin, W. A. Atkinson, I. V. Gornyi, P. J. Hirschfeld and D. V. Khveshchenko, Phys. Rev. Lett. 86, 5982 (2001).

3 A. Altland, Phys. Rev. B 65, 104525 (2002).

4 A. V. Balatsky, M. I. Salkola and A. Rosengren, Phys. Rev. B 51, 15547 (1995); M. I. Salkola, A. V. Balatsky and D. J. Scalapino, Phys. Rev. Lett. 77, 1841 (1996).

${ }^{5}$ I. Adagideli, P. M. Goldbart, A. Shnirman and A. Yazdani, Phys. Rev. Lett. 83, 5571 (1999).

${ }^{6}$ Of course, the mechanism for creating low-energy states invoked in the present Paper does not specifically require dwave superconductivity. All that is required is that the pair potential be real (or can be made so by a suitable gauge transformation) and that it possess regions of momentum orientation over which it has opposing signs.

7 A. Yazdani, C. M. Howald, C. P. Lutz, A. Kapitulnik and D. M. Eigler, Phys. Rev. Lett. 83 (1999) 176.

8 Similar states have also been observed in the case of native defects in: E. Hudson, S. H. Pan, A. K. Gupta, K.-W. Ng and J. C. Davis, Science 285, 88 (1999).

9 E. Witten, Nucl. Phys. B 188, 513 (1981).

10 P. Salomonson and J.W. Van Holten, Nucl. Phys. B 196, 509 (1982).

11 See e.g., G. Junker, Supersymmetric Methods in Quantum and Statistical Physics (Springer, Berlin, 1996).

12 F. J. Dyson, Phys. Rev. 92, 1331 (1953).

13 C. Chamon and C. Mudry, Phys. Rev. B. 63, 100503 (2001).
14 D. E. Sheehy, I. Adagideli, P.M. Goldbart and A. Yazdani, Phys. Rev. B 64, 224518 (2001).

15 A. F. Andreev, Zh. Eksp. Teor. Fiz. 46, 1823 (1964) [Sov. Phys. JETP 19, 1228 (1964)].

16 D. Waxman and K. D. Ivanova-Moser, Ann. Phys. 226, 271 (1993).

17 A. Comtet, J. Desbois and C. Monthus, Ann. Phys. 239, 312 (1995).

18 L. Balents and M. P. A. Fisher, Phys. Rev. B 56, 12970 (1997).

19 See, e.g., L. Bartosch, Ann. Phys. (Leipzig) 10, 799 (2001).

20 Higher-order tunneling matrix elements are small, relative to the leadinforder contributions, provided that $\int_{s_{n}}^{s_{n+1}}|\Delta(s)| d s \gg 10$, i.e., that the coherence length is small compared with the typical impurity spacing. In addition, we note that, as $\hat{H}$ may be made purely off-diagonal by a suitable unitary transformation, the tunneling amplitude between next-nearest neighbors vanishes identically.

21 The related task of computing tunnelling corrections to the ground state of a SUSY Hamiltonian having multiple degenerate minima using instanton methods has been considered in Ref. 10 .

22 T. P. Eggarter and R. Riedinger, Phys. Rev. B 18, 569 (1978).

23 G. Theodorou and M. H. Cohen, Phys. Rev. B 13, 4597 (1976).

24 We suspect that the result for the DOS presented here will continue to hold not solely in the setting of uncorrelated hopping matrix elements $\left\{t_{n}\right\}$ but also for correlations of finite range. 